\title{
Swing the balance
}

Cell mass and volume fluctuations are not only linked to basic cellular processes such as cell growth but can also be an important indicator of diseases such as viral infections. Assessing the mass of an individual adherent cell with high temporal resolution has not yet been possible. Daniel Müller and colleagues have developed a picobalance that can measure the mass of a single cell with a temporal resolution high enough to follow mass fluctuations on the millisecond timescale, as they report in Nature.

Cell volume and mass are interconnected, and the regulation of cell size is at the heart of key cellular events, such as growth, division and survival. The mass of a cell is mainly determined by the amount of water, proteins and nutrients it contains and is therefore linked to cell metabolism and gene expression. It is well known that pathogen infections lead to changes in cell mass and shape; however, owing to a lack of adequate methods for measuring the mass of adherent cells with high sensitivity, until now, such alterations could only be assessed by visual examination.

Daniel Müller and colleagues have employed a silicone microcantilever mounted on an inverted optical microscope that can operate in physiological conditions. A single cell can be attached to the cantilever by functionalizing its surface with extracellular matrix proteins and by applying a very small force. The attachment of a cell to the cantilever changes the effective mass of the cantilever and therefore its resonance frequency. A low-power blue laser focused on the base of the cantilever generates extremely small cantilever oscillations, which makes the cantilever even more sensitive to effective mass alterations. The changes in cantilever movement are measured by a second infrared laser and can be used to analyse cell mass with high sensitivity. The optical microscope enables the correct placement of the cell onto the cantilever and concurrent fluorescent imaging. "Together with our picobalance we have also developed an environmental chamber that provides cell culture conditions during the experiments. The whole set-up, which is fully compatible with modern optical microscopy approaches, allows monitoring of the cell mass over several days," explains Müller.

Using this set-up, the researchers determined the mass of fibroblasts and HeLa cells and observed that dynamic mass fluctuations happened within a few seconds. Through perturbing either water transport across the cell membrane, cellular metabolism or ATP synthesis, Müller and colleagues demonstrated that the mass fluctuations depend on water exchange and energy supply. "The high mass and temporal resolution of our new technology, the picobalance, allowed us to discover that the mass of animal cells fluctuates within seconds throughout the cell cycle. Perturbation experiments link these mass fluctuations to basic cellular processes such as ATP synthesis and water transport," comments Müller.

The cells were then infected with a vaccinia virus by attaching an infected cell to the cantilever and placing it on top of a healthy cell. This approach enabled virus transfection from one cell to the other. Cell mass monitoring over 40 hours revealed that the mass remained constant in the infected cell despite the intracellular production of new viruses. By contrast, the mass of healthy cells continuously increased. "The vaccinia virus arrests cell growth and cycle progression, whereas the healthy cells show fluctuating mass until cell death," explains Müller. "Understanding how cells regulate mass and how pathogens affect this regulation might provide new insights into physiological processes and infectious diseases."

The researchers are currently developing new strategies for attaching cells to the cantilever; for example, by transferring their technology to micropipettes that hold single cells. This would enable them to detect the mass of cells progressing through mitosis. They further plan to adapt the technology to follow the mass of larger samples, such as bacteria, yeast and organoids.

Christine-Maria Horejs

ORIGINAL ARTICLE Martínez-Martín, D. et al.

Inertial picobalance reveals fast mass fluctuations in mammalian cells. Nature 550, 500-505 (2017)
The high mass and temporal

resolution

of our new

technology, the picobalance, allowed us to discover that the mass of animal cells fluctuates within seconds throughout the cell cycle.

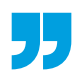

